1

2

3

4

5

6

7

8

9

10

11

12

13

14

15 Correspondence

16

17

18

19 Conflicts of Interest

20 The authors declare no conflicts of interest.

\section{Source of Funding}

22 This work was supported by a Discovery grant awarded from the Natural Sciences and

23 Engineering Research Council of Canada [RGPIN-2017-06969] as well as the NSERC-Sonova

24 Senior Industrial Research Chair [IRC-537355-18], both awarded to F. A. Russo. 


\section{Abstract}

26 Objectives: Understanding speech in noise can be highly effortful. Decreasing the signal-to-

27 noise ratio (SNR) of speech increases listening effort, but it is relatively unclear if decreasing the

28 level of semantic context does as well. The current study used functional near-infrared

29 spectroscopy (fNIRS) to evaluate two primary hypotheses: (1) listening effort (operationalized as

30 oxygenation of the left lateral PFC) increases as the SNR decreases and (2) listening effort

31 increases as context decreases.

32 Design: Twenty-eight younger adults with normal hearing completed the Revised Speech

33 Perception in Noise (R-SPIN) Test, in which they listened to sentences and reported the final

34 word. These sentences either had an easy SNR $(+4 \mathrm{~dB})$ or a hard SNR $(-2 \mathrm{~dB})$, and were either

35 low in semantic context (e.g., "Tom could have thought about the sport") or high in context (e.g.,

36 "She had to vacuum the rug"). PFC oxygenation was measured throughout using fNIRS.

37 Results: Accuracy on the R-SPIN Test was worse when the SNR was hard than when it was

38 easy, and worse for sentences low in semantic context than high in context. Similarly,

39 oxygenation across the entire PFC (including the left lateral PFC) was greater when the SNR was

40 hard, and left lateral PFC oxygenation was greater when context was low.

41 Conclusions: These results suggest that activation of the left lateral PFC (interpreted here as

42 reflecting listening effort) increases to compensate for acoustic and linguistic challenges. This

43 may reflect the increased engagement of domain-general and domain-specific processes

44 subserved by the DLPFC (e.g., cognitive control) and IFG (e.g., predicting the sensory

45 consequences of articulatory gestures), respectively. 


\section{Introduction}

When listening conditions are challenging, such as in the presence of background noise,

50

51

52

53

54

55

56

57

58

59

60

61

62

63

64

65

67

68

69

70

listeners may need to work especially hard as they attempt to maintain levels of speech understanding. This process is known as listening effort, defined as "the deliberate allocation of mental resources to overcome obstacles in goal pursuit when carrying out a [listening] task" (Pichora-Fuller et al. 2016, p. 10S). Listening effort is of considerable interest to clinicians, as people with hearing loss may experience elevated listening effort despite normal pure-tone thresholds (Plack et al. 2014). Such elevated listening effort can lead to cognitive consequences such as reduced memory for what you are listening to (McCoy et al. 2005) as well as social consequences such as withdrawal from situations in which listening is effortful (Pichora-Fuller et al. 2015). These consequences have motivated researchers to investigate the conditions under which listening effort is elevated. In the current study, we use a silent neuroimaging method known as functional near-infrared spectroscopy (fNIRS) to measure listening effort. In particular, we consider how listening effort is affected by the level of background noise (acoustic challenge) and the level of semantic context (linguistic challenge).

\section{Listening Effort and Background Noise}

The factor most often shown to affect listening effort is the acoustic challenge of speech. This is frequently dictated by the level of background noise relative to target speech, or signal-to-noise ratio (SNR), where a decreasing SNR indicates increasing noise relative to speech. Comprehending speech in noise (e.g., competing talkers) presents a unique set of challenges to listeners. For instance, energetic masking occurs when target speech and noise fall within the same critical band, rendering target speech less available due to physical interactions that are taking place in the periphery (Fletcher \& Galt 1950). Another form of masking is 
71 informational, broadly defined as any form of masking that is not energetic (Durlach 2006). This

72 is usually characterized by interference from sounds that are similar to the target, thus making

73 them difficult to separate perceptually (Brungart 2001).

74 These unique sources of masking place a load on cognitive processes that support

75 stream segregation to separate target speech from the noise (e.g., based on spectral cues),

76 selective attention to the speech over the noise, and perceptual closure to "fill in" any portions of

77 the speech that were occluded by the noise (e.g., based on linguistic or general world knowledge;

78 Mattys et al. 2012; Johnsrude \& Rodd 2016). This processing load causes listening to become

79 more effortful, as has been demonstrated using several types of measures (for a review of

80 measures, see McGarrigle et al. 2014). These include subjective (i.e., self-reported listening

81 effort; Rudner et al. 2012; Johnson et al. 2015), behavioural (e.g., reaction time, secondary-task

82 performance; Sarampalis et al. 2009; Houben et al. 2013; Wu et al. 2016), and peripheral

83 physiological measures (e.g., pupil dilation; Kramer et al. 1997; Zekveld et al. 2010), all of

84 which report increased listening effort as the SNR decreases. However, once the SNR becomes

85 sufficiently challenging, listeners are increasingly likely to disengage, leading to a decrease in

86 listening effort (Pichora-Fuller et al. 2016; Peelle 2018; Herrmann \& Johnsrude 2020).

Important questions have been raised about the above measures of listening effort. For

88 instance, these measures do not usually correlate with one another, suggesting that they may not

89 measure the same construct (Strand et al. 2018; Alhanbali et al. 2019; Strand et al. 2020).

90 Another method to study listening effort involves neuroimaging, which offers a more direct look

91 at the recruitment of cognitive resources to support listening (Evans \& McGettigan 2017; Peelle

92 2018; Herrmann \& Johnsrude 2020). In contrast, subjective, behavioural, and peripheral

93 physiological measures of listening effort are more likely to be confounded by internal or 
94 external factors (e.g., perceived task performance in the case of self-reported listening effort;

95 Moore \& Picou 2018; for discussion, see Rovetti et al. 2019). Thus, it is unsurprising that a

96 review by Ohlenforst et al. (2017) concluded that neural measures of listening effort-

97 specifically functional magnetic resonance imaging (fMRI) and electroencephalography (EEG),

98 the only neuroimaging methods considered — may be uniquely suited to detect increases in

99 listening effort resulting from hearing loss. Given these strengths, many recent studies of

100 effortful listening have opted to employ neuroimaging methods.

\section{The Neural Basis of Listening Effort}

Effortful listening in noise is marked by increased activation of two primary brain networks (Alain et al. 2018; Peelle 2018). The first network is the domain-general multiple-

104 demand system (Duncan 2010; Camilleri et al. 2018), which can be divided into two subnetworks (Dosenbach et al. 2008): the cingulo-opercular network, composed of dorsal cingulate,

106 inferior frontal, and anterior insula regions (Dosenbach et al. 2006; Eckert et al. 2009); and the

107 frontoparietal network, composed of brain areas along the dorsolateral prefrontal cortex

108 (DLPFC) and intraparietal sulcus (Ptak 2011; Marek \& Dosenbach 2018). The second network to 109 support speech perception in noise is a domain-specific speech processing network specialized 110 for speech and language, including the inferior frontal gyrus (IFG), premotor cortex, and inferior 111 parietal lobule (Hickok \& Poeppel 2007; Rauschecker \& Scott 2009). When it comes to the multiple-demand system, the cingulo-opercular network becomes

113 active when we are motivated to understand speech, and when cognitive control (i.e., goal-

114 directed behaviour) is needed to monitor and optimize our performance (Vaden et al. 2013;

115 Vaden et al. 2015). This network may then signal activation of the fronto-parietal network

116 (Botvinick et al. 2004), including the DLPFC, which directs attention to relevant information and 
117 suppresses irrelevant information (Eckert et al. 2016). Numerous fMRI studies have previously

118 found that the DLPFC becomes more active as the SNR decreases (Sharp et al. 2006; Eckert et

119 al. 2008; Wong et al. 2008), as have positron emission tomography studies (Salvi et al. 2002;

120 Scott et al. 2004). EEG studies have also investigated listening in noise, such as Wisniewski et

121 al. (2015), who found that frontal alpha power increased as the SNR decreased.

As for the speech processing network, these brain areas are theorized to perform several

123 functions to support speech perception in noise. First, they may contribute verbal working

124 memory, allowing unclear speech to be held in memory as it undergoes further processing

125 (Fedorenko et al. 2012; Rönnberg et al. 2013; Peelle \& Wingfield 2016; Peelle 2018). Second,

126 because this network is also responsible for speech production, it may predict the sensory

127 consequences of articulatory gestures in such a way that it helps to disambiguate what is being

128 heard (Hervais-Adelman et al. 2012; Du et al. 2016; Skipper et al. 2017). Third and finally, the

129 IFG in particular likely contributes higher-level linguistic processing, such as drawing on prior

130 linguistic and world knowledge to aid in perceptual closure (Davis \& Johnsrude 2003; Wild et al.

131 2012; Johnsrude \& Rodd 2016). Numerous fMRI studies have found that the (usually left) IFG

132 becomes more active as the SNR decreases (Adank et al. 2012; Vaden et al. 2013; Du et al.

133 2014), which was also confirmed by a meta-analysis from Alain et al. (2018).

134 Listening Effort and Semantic Context

In addition to SNR and other factors that change the acoustic challenge of speech, the

136 linguistic content of speech can also affect processing load. One such linguistic factor is the level

137 of semantic context present to facilitate speech perception. For instance, in a sentence such as

138 "The boy likes to look at the snow," little semantic context is present to help the listener predict

139 the final word. Thus, if the final word is unclear (e.g., due to occlusion from background noise), 
140 the listener has to consider thousands of candidate words, which together comprise the lexical

141 search space (Wagner et al. 2016b). The larger the lexical search space, the more mental

142 conflict — or lexical competition — is experienced as the listener determines the correct

143 interpretation (Luce \& Pisoni 1998; Dahan et al. 2001). In contrast, in the sentence "The boy

144 rolled around in the snow," the final word is highly predictable based on the context of the

145 sentence, as there are only so many candidates that are semantically coherent (e.g., grass, mud,

146 sand). Thus, semantic context reduces the lexical search space and, as a result, lexical

147 competition (McClelland \& Elman 1986; Strand et al. 2013).

Pupillometry studies have reported that overcoming lexical competition requires

149 considerable listening effort (Kuchinsky et al. 2013; Wagner et al. 2016b). It is thus sensible to

150 assume that decreased semantic context, which would increase lexical competition, would also

151 lead to increased listening effort, likely by increasing the load on higher-level linguistic

152 processes that support "filling in" occluded speech (Johnsrude \& Rodd 2016). However, the

153 literature is mixed on this question. Numerous studies have found that listening effort is

154 increased when context is decreased, including studies of self-reported listening effort (Johnson

155 et al. 2015; Holmes et al. 2018), dual-task performance (Pichora-Fuller et al. 1995; Johnson et al.

156 2015), and pupil dilation (Winn 2016; Lau et al. 2019; Kadem et al. 2020). In contrast, other

157 studies have found that listening effort is not influenced by context, including two studies of

158 dual-task performance (Tun et al. 2009; Desjardins \& Doherty 2014) and one of pupil dilation

159 (Borghini \& Hazan 2020). One study even found that self-reported listening effort decreases

160 when context is lower (Lau et al. 2019). It should be noted that in addition to the exact measures

161 used to probe listening effort, these studies varied in their populations and stimuli, meaning their 
162 results are difficult to compare. Nevertheless, no one of these factors seems to predict whether or 163 not context affects listening effort.

Only a handful of studies have manipulated the semantic context of speech while measuring the brain. For instance, Davis et al. (2011) found that the left IFG was more active when participants listened to sentences presented in speech envelope and spectrum noise with low context than high context, at least up until the point where the SNR became very hard. Zekveld et al. (2012) described similar left IFG activation when participants listened to sentences (both in clear and speech-shaped stationary noise) that were preceded by semantically-unrelated cue words rather than semantically-related cue words. However, these two studies contradict Obleser et al. (2007), who reported greater left IFG and DLPFC activation when participants listened to noise-vocoded sentences with high context than low context. As with studies involving non-neural measures, the results of these neuroimaging studies are difficult to compare given the diversity of methods employed. Thus, even when it comes to the neural assessment of listening effort, it is unclear whether decreased context increases listening effort.

\section{Functional Near-Infrared Spectroscopy}

Apart from fMRI, another neuroimaging method that may be suitable to study listening effort is fNIRS. fNIRS works by using light sources to emit near-infrared light into the cortex. On the basis of how much of that light is scattered back and measured by light detectors, the concentration of oxygen in the cortex (i.e., brain activity) can be inferred (Izzetoglu 2012). As with fMRI, fNIRS is sensitive to the hemodynamic response, which may take up to $10 \mathrm{~s}$ or even longer to evolve after task onset (Miezin et al. 2000). For many years, fNIRS measurement of the PFC has been used to effectively assess cognitive effort and workload during a variety of tasks (Ayaz et al. 2012; Harrivel et al. 2013; Fishburn et al. 2014). Thus, it is unsurprising that fNIRS 
185 has recently been used to measure listening effort, operationalized as the recruitment of

186 prefrontal brain areas that are associated with effortful listening (Wijayasiri et al. 2017;

187 Lawrence et al. 2018; Rowland et al. 2018; Rovetti et al. 2019; Zhou et al. accepted). Indeed,

188 fNIRS is well-suited to study speech and language, as it is silent (for discussion of fMRI and

189 scanner noise, see Peelle et al. 2010), tolerant of motion artefacts (Metzger et al. 2017), and

190 resistant to interference from nearby electronic equipment (e.g., hearing assistive devices; Van

191 de Rijt et al. 2016; Rovetti et al. 2019).

192 One of the first fNIRS studies to measure listening effort was Wijayasiri et al. (2017),

193 who found that the left IFG was most active when participants were presented with noise-

194 vocoded speech that was challenging to perceive accurately but still intelligible (as opposed to

195 clear or unintelligible speech), but only when they attended to this speech rather than a distractor.

196 These results replicated earlier findings obtained with fMRI (Wild et al. 2012). More recently,

197 Rowland et al. (2018) monitored the PFC of participants while listening to complex, naturalistic

198 stimuli ranging in a variety of acoustic features, including SNR (which ranged from -16 dB to

$199+25 \mathrm{~dB})$. Although PFC activation was found to be significantly correlated across participants,

200 there was no effect of SNR on activation in any part of the PFC. This could be due to the

201 considerable variability of the stimuli (e.g., the environments in which the noise was recorded).

202 This was the only fNIRS study to assess the effect of SNR on listening effort, and thus no fNIRS

203 study has found a relationship between these variables. In addition, no fNIRS study has assessed

204 whether semantic context (or any other linguistic factors) affects listening effort.

\section{The Current Study}

206 To address the shortcomings in the literature described above, we recruited 28 younger

207 adults with normal hearing to complete the Revised Speech Perception in Noise (R-SPIN) Test, 
208 in which participants listen to sentences presented among background noise and reported the

209 final word. Two independent variables were manipulated: the SNR ( $+4 \mathrm{~dB}$ [easy] or $-2 \mathrm{~dB}$

210 [hard]) and the level of semantic context (low or high). We used fNIRS to measure oxygenation

211 in four subregions of the PFC, defined by their position along the coronal plane: the left lateral

212 PFC, left medial PFC, right medial PFC, and right lateral PFC. The lateral PFC subregions

213 includes the DLPFC and IFG, while the medial PFC subregions includes the frontopolar cortex

214 (Liu et al. 2017). Left lateral PFC oxygenation was our operationalization of listening effort, as

215 the left DLPFC and IFG tend to be recruited in response to acoustic and linguistic challenge

216 (Scott et al. 2004; Davis et al. 2011; Du et al. 2014). We predicted that (1) left lateral PFC

217 oxygenation (i.e., listening effort) would increase as the SNR decreased and (2) left lateral PFC

218 oxygenation would increase as semantic context decreased.

Materials and Methods

\section{Participants}

221

Data are reported for 28 normal-hearing younger adults, who were recruited from

222 undergraduate psychology courses and the community. Participants had ages ranging from 18 to

22335 years $(M=24.1, S D=5.07)$ and pure-tone averages (PTA; measured as the binaural average

224 at $500,1000,2000$, and $4000 \mathrm{~Hz})$ ranging from 0.63 to $16.88 \mathrm{~dB} \mathrm{HL}(M=8.03, S D=4.18)$,

225 indicating that all had normal hearing. Data were collected from five additional participants, but

226 they were replaced for the following reasons: having a PTA greater than $25 \mathrm{~dB} \mathrm{HL}(n=1)$; being

227 older than 35 years of age $(n=1)$; learning English after the age of seven $(n=1)$; and

228 experimenter error resulting in their data not being saved $(n=2)$. The sample size was selected

229 based on a power analysis done in $\mathrm{R}$ version 4.0.3 ( $\mathrm{R}$ Core Team 2020) with the package

230 "Superpower" version 0.1.0 (Lakens \& Caldwell 2019). This number was sufficient to detect 
231 effects of SNR and context on left lateral PFC oxygenation with $80 \%$ power, assuming a

232 moderate correlation across conditions $(r=.5)$ and small-medium effect sizes $(d=0.4$; Zekveld

233 et al. 2012; Johnson et al. 2015; Holmes et al. 2018; Lau et al. 2019).

234 Design

235

The experiment was based on a three-factor within-subject design. The independent

236 variables were PFC subregion (left lateral, left medial, right medial, or right lateral), R-SPIN

237 Test SNR (+4 dB [easy] or $-2 \mathrm{~dB}$ [hard]), and R-SPIN Test semantic context (low or high). The

238 dependent variables were R-SPIN Test accuracy (expressed as a proportion) and oxygenation

239 (expressed in arbitrary units). Listening effort was operationalized as oxygenation of the left

240 lateral PFC. No other measures of listening effort were used. The study was approved by the

241 Research Ethics Board at Ryerson University (protocol number 2017-187).

242 Tasks and Measures

243 R-SPIN Test. Stimuli consisted of 100 clips of spoken sentences obtained from lists 5

244 and 7 of the R-SPIN Test (Bilger et al. 1984). These clips ranged from 9.03 to $12.18 \mathrm{~s}$ in duration

$245(M=10.54, S D=0.46)$. Participants were instructed to listen to these clips and repeat back the

246 final word of each sentence. If they could not identify the final word, they were instructed to

247 guess or say "pass." The speech was spoken by a male with a North American accent, and the

248 background noise - playing throughout the entire clip — was 12-talker babble (six male and six

249 female voices reading aloud). Each clip began with a spoken stimulus number (e.g., "Number

250 one") to aid the later review of responses by the experimenter. After that number, the sentence

251 itself was spoken, and then a few seconds were present before the end of the clip to give

252 participants a chance to repeat back the final word. The sentences either contained a low or high 
253 degree of contextual information that would help to identify the final word of each sentence (e.g.,

254 "Tom could have thought about the sport" or "She had to vacuum the rug").

The 100 sentences were divided into two 50-sentence lists (one low context, one high

256 context). Participants were presented with a list of one context level followed by a list of the

257 other context level (e.g., low context followed by high context). In addition, the first 25

258 sentences of both lists had one SNR and the last 25 sentences of both lists had the other SNR

259 (e.g., easy SNR followed by hard SNR within each of the two lists). Thus, each participant was

260 presented with four blocks of 25 sentences, with each block just over $250 \mathrm{~s}$. The two context

261 orders and two SNR orders were counterbalanced across participants. This gave rise to four

262 block orders, each completed by seven participants. For example, a participant may have

263 completed the blocks in the following order: (1) easy SNR/high context, (2) hard SNR/high

264 context, (3) easy SNR/low context, and (4) hard SNR/low context.

265 fNIRS. Figure 1 shows the layout of the fNIR Imager 1100 (fNIR Devices, LLC,

266 Potomac, USA), the apparatus with which we collected PFC oxygenation data. This is a 16-

267 channel continuous wave optical neuroimaging system, which emits near-infrared light of 730

$268 \mathrm{~nm}$ and $850 \mathrm{~nm}$ into the forehead. Cognitive Optical Brain Imaging Studio version 1.4 (Ayaz \&

269 Onaral 2005) was the platform used to control the acquisition of light intensity data, completed at

270 a sampling rate of $2 \mathrm{~Hz}$. In each channel, oxygenation was calculated as the difference between

271 the concentrations of oxygenated and deoxygenated hemoglobin ( $\mathrm{HbO}-\mathrm{HbR})$. This composite

272 measure - known as the change in cerebral oxygen exchange — is highly sensitive, as it captures

273 both the increase in $\mathrm{HbO}$ and the decrease in HbR that accompanies brain activation (Kato et al.

274 1993; Liang et al. 2016; Saleh et al. 2018; Pinti et al. 2019). Left lateral PFC oxygenation was

275 calculated as the average of oxygenation in channels 1-4, left medial PFC oxygenation channels 
276 5-8, right medial PFC oxygenation channels 9-12, and right lateral PFC 13-16, all as in prior

277 studies with a similar apparatus (Aranyi et al. 2015; Cavazza et al. 2015; Liang et al. 2016;

278 Montgomery et al. 2017; Anderson et al. 2018; Rovetti et al. 2019, 2021).

279 Listening effort was operationalized as oxygenation of the left lateral PFC, as this region

280 has previously been found to become more active in response to acoustic and linguistic challenge

281 (Scott et al. 2000; Wong et al. 2008; David et al. 2011; Zekveld et al. 2012; Du et al. 2014;

282 Wijayasiri et al. 2017). Left lateral PFC activation also decreases once task demands exceed the 283 listeners' cognitive capacity (Davis \& Johnsrude 2003; Wild et al. 2012), as is also true of 284 listening effort (Peelle 2018; Herrmann \& Johnsrude 2020). Our previous fNIRS studies to 285 successfully measure cognitive effort and listening effort have operationalized it in the same way 286 (Rovetti et al. 2019, 2021). This operationalization is meant to align with the definition of 287 listening effort being used: "the deliberate allocation of mental resources to overcome obstacles 288 in goal pursuit when carrying out a [listening] task" (Pichora-Fuller et al. 2016, p. 10S). In other 289 words, listening effort is being defined as the recruitment of cognitive resources to support 290 listening, whereas other studies may instead be interested in the subjective experience of 291 listening being effortful or some other definition (Herrmann \& Johnsrude 2020; Strand et al. 292 2020). 

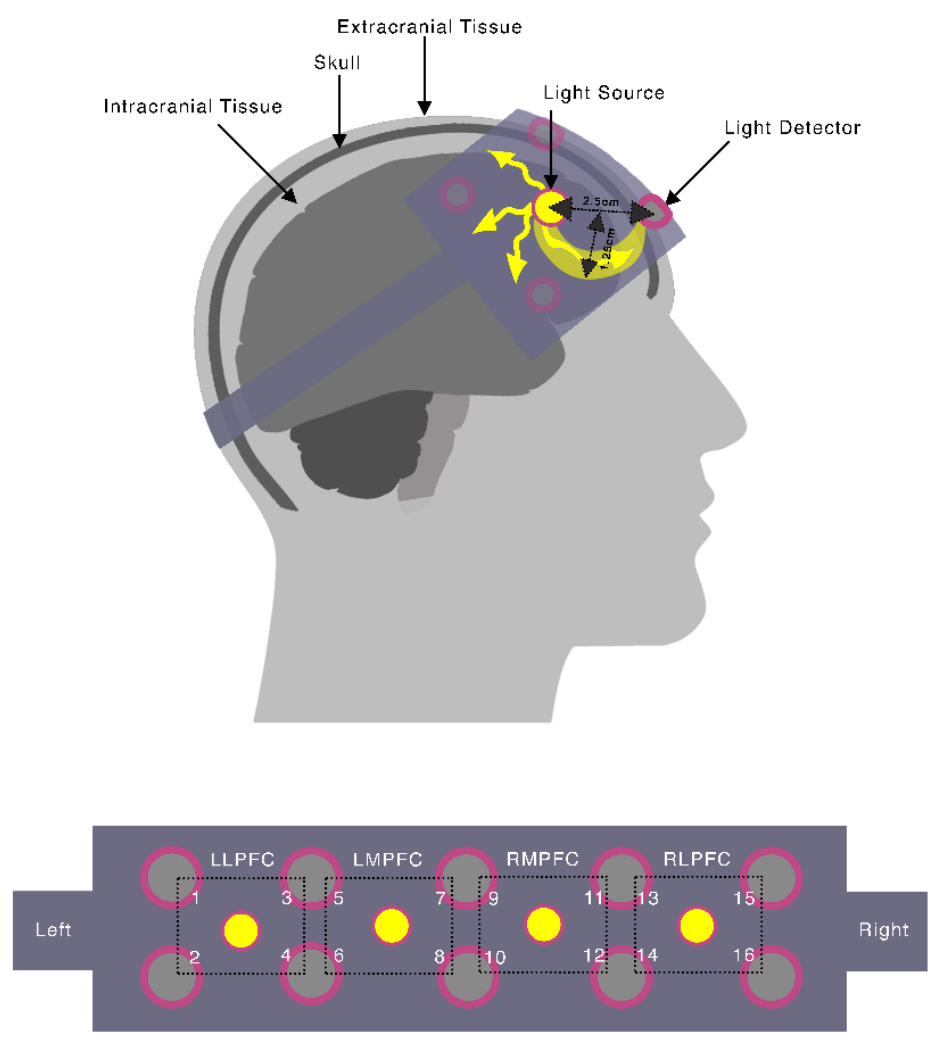

294 Figure 1. The fNIRS sensor pad was secured to participants' foreheads (top). This sensor pad 295 had 10 light sources (grey circles) and four light detectors (yellow circles), which defined 16 296 channels in which oxygenation was measured (numbered in white; bottom). These 16 channels 297 were grouped into four PFC subregions (black boxes): LLPFC = left lateral PFC, LMPFC = left 298 medial PFC, RMPFC = right medial PFC, RLPFC = right lateral PFC. Figure adapted from 299 Rovetti et al. (2019).

300 Procedure

Upon arrival in the lab, participants were given a detailed description of the study, 302 provided written informed consent, and completed prescreen and demographic questionnaires. 303 Participants were then seated in a double-walled sound-attenuated chamber where they 304 completed pure-tone audiometric testing using a Grason-Stadler GSI 61 audiometer. They next 
put on Sennheiser HD 518 headphones and were given an explanation of the task. Windows Media Player was then used to play two practice R-SPIN Test sentences, which were presented at $72 \mathrm{~dB}$ SPL and with no background noise. Once participants expressed an understanding of the task, the fNIRS sensor pad was affixed to and centred on their foreheads above the eyebrows, aligned with the FpZ location according to the international 10-20 system (Homan et al. 1987). Adjustments were then made to the sensor pad placement and device settings (e.g., detector gain) to optimize signal quality (for details, see Rovetti et al. 2019, 2021).

Before the very first block, a 10-s baseline was recorded, over which participants were instructed to relax and remain still. Windows Media Player was used to present the R-SPIN Test blocks, with the order determined by the counterbalancing scheme (for details, see Tasks and Measures). A block design was chosen to reduce the influence of noise unrelated to brain activity (Cui et al. 2011). At the start of each block, the experimenter pressed a key to manually place a "marker" in the data acquisition platform. This gave a timestamp to the start of the block and allowed it to later be selected and exported at the data processing stage. Once a block began, the rate of stimulus presentation was automatic, with the next clip immediately following the last. Speech was presented at $72 \mathrm{~dB}$ SPL across all blocks and the level of noise was determined by the SNR. A one-way audio monitor was used by the experimenter to listen to and score the accuracy of participants' responses in real time, and their responses were also recorded for later review. There was no set break between blocks; once the next block was prepared by the experimenter (approximately $10 \mathrm{~s}$ ), the participant could begin the next block whenever they were ready (on average, there was approximately $20 \mathrm{~s}$ between the end of one block and the start of the next). After completing all blocks, participants were debriefed. 


\section{Data Processing}

Using fNIRSoft Professional version 4.6 (Ayaz 2010), the following pre-processing steps were applied to the light intensity data: (1) finite impulse response linear phase low-pass filtering (order $=20$, cut-off frequency $=0.1 \mathrm{~Hz}$ ) to reduce physiological and equipment noise (e.g., heartbeat), (2) removal of motion artefacts using the sliding-window motion artefact removal algorithm (window size $=10 \mathrm{~s}$, upper threshold $=0.025 \mathrm{~nm}$, lower threshold $=0.003 \mathrm{~nm}$; Ayaz et al. 2010), (3) manual rejection of channels whose raw signal values fell below $400 \mathrm{mV}$ (suggesting light detector obstruction) or exceeded $4000 \mathrm{mV}$ (suggesting light detector saturation), (4) conversion of light intensity data to oxygenation data using the modified BeerLambert law (Kocsis et al. 2006), (5) and baseline correction of each channel according to the mean of that channel's 10 -s baseline recorded at the start of the experiment.

In post-processing, channels were also rejected if more than $50 \%$ of their data were missing across all conditions (e.g., due to motion artefacts). The mean number of channels rejected per participant, of 16 in total, was $2.64(S D=1.85)$. Means were similar among the four PFC subregions, although the right lateral PFC had the highest rate of channel rejection (left lateral: $M=0.61$; left medial: $M=0.50$; right medial: $M=0.46$; right lateral: $M=1.07)$. When a channel was rejected for a participant, it was rejected across all conditions. For each block, oxygenation in each channel was calculated as the mean from $10 \mathrm{~s}$ into the block (to allow the hemodynamic response a chance to evolve in response to the task) until $250 \mathrm{~s}$ after the start of the block. Oxygenation in each PFC subregion was then calculated by averaging together oxygenation from the four channels that comprise it, or fewer in cases where channels were rejected. These pre- and post-processing steps are almost identical to Rovetti et al. $(2019,2021)$, with the only difference being that linear detrending was not used in the current study. With all 
351 participants completing two conditions of one context level followed by two conditions of the

352 other context level, using linear detrending to eliminate signal drift over time would have also

353 eliminated the effect of context, as it also tied to time.

354 Statistical Analyses

355 All statistical analyses were done using R version 4.0.3. Analysis of variance (ANOVA)

356 was conducted using the package "ez" version 4.4.0, with Greenhouse-Geisser adjustment of

357 degrees of freedom applied to effects that failed Mauchly's test of sphericity. These analyses

358 were used to assess the effects of SNR and semantic context on accuracy; and SNR, context, and

359 PFC subregion on oxygenation. Analyses of oxygenation considered all trials, including those in

360 which participants answered "pass," which represented $8.11 \%$ of all responses. Effect sizes for

361 follow-up paired-samples $t$-tests were calculated using the package "lsr" version 0.5. The

362 package "rmcorr" version 0.4.1 (Bakdash \& Marusich 2017) was used to conduct exploratory

363 repeated-measures correlational analyses, a technique that calculates the within-subject

364 association between two variables, equivalent to multilevel modelling with intercepts (but not

365 slopes) allowed to vary randomly across participants. In particular, these analyses assessed

366 whether, as accuracy changed on a within-subject basis from condition to condition, oxygenation

367 in any PFC subregion changed along with it. Figure 1 was created in Adobe Photoshop version

368 20.0; Figures 2 and 3 were created in R using the packages "tidyverse" version 1.3.0, "grid"

369 version 4.0.3, and "gridExtra" version 2.3; and Figure 4 was created in MATLAB version 9.9.

Results

371

Data and code for the analyses reported can be found at the following link:

372 https://osf.io/pavtd/ 


\section{$374 \quad$ R-SPIN Test Accuracy}

375

376

377

378

379

380

381

382

383

384

385

386

Figure 2 shows accuracy as a function of SNR and context (averaged across participants). A repeated-measures $2(\mathrm{SNR}) \times 2$ (context) ANOVA found significant main effects of SNR

$\left(F[1,27]=334, p<.001, \eta_{\mathrm{G}}{ }^{2}=.45\right)$ and context $\left(F[1,27]=159, p<.001, \eta_{\mathrm{G}}{ }^{2}=.50\right)$ on accuracy, with accuracy worse when the SNR was hard than when the SNR was easy, and worse when context was low than when context was high. There was also a significant interaction between $\mathrm{SNR}$ and context on accuracy $\left(F[1,27]=22.1, p<.001, \eta_{\mathrm{G}}{ }^{2}=.076\right)$. Paired-samples $t$-tests were used to compare the effect of context on accuracy at each SNR. This found that accuracy was significantly worse when context was low both when the SNR was easy (low context: $M=0.69$, $S D=0.13$; high context: $M=0.90, S D=0.13 ; t[27]=-10.7, p<.001, d=2.02)$ and when the SNR was hard (low context: $M=0.34, S D=0.11$; high context: $M=0.72, S D=0.20 ; t[27]=-$ $10.4, p<.001, d=1.96)$. The interaction was driven by the larger effect of context on accuracy when the SNR was hard (0.38) than when the SNR was easy (0.21). 


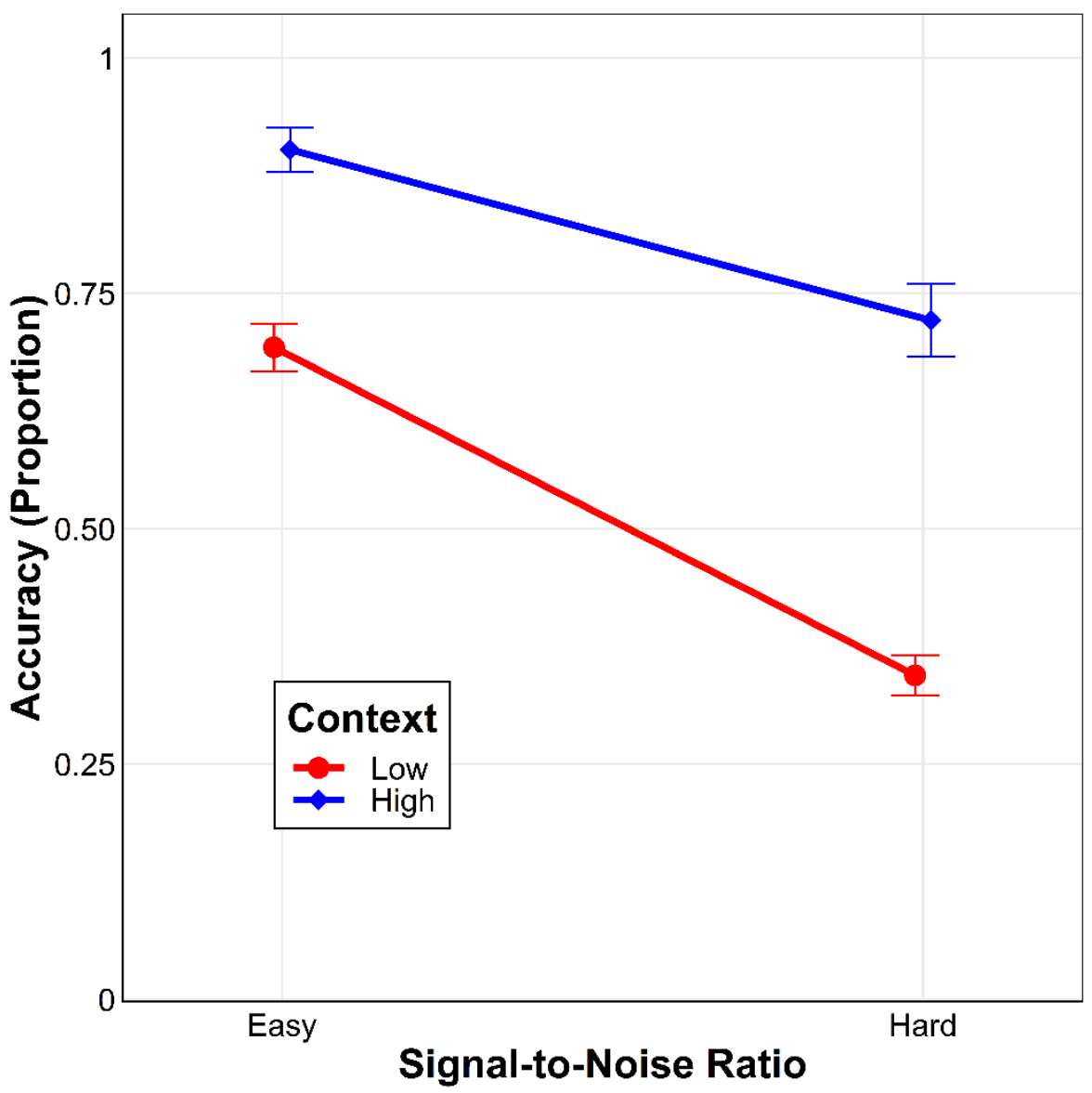

Figure 2. Accuracy was better when the SNR was easy than when the SNR was hard, and was better when semantic context was high than when the context was low. The effect of SNR was greater when context was low. Error bars show the standard errors of the mean.

Recall that half of our sample completed the low-context conditions followed by the high-context conditions, and the other half high-context followed by low-context. Since the order in which these context levels were completed could have affected the strategy that participants used, we also explored effect of context order (low-high or high-low) on accuracy using a mixed $2(\mathrm{SNR} ;$ within-subject $) \times 2$ (context; within-subject $) \times 2$ (context order; between-subject $)$ ANOVA. However, there was no effect of context $\times$ context order $\left(F[1,26]=0.68, p=.42, \eta_{\mathrm{G}^{2}}{ }^{2}=\right.$ $.005)$ or any other significant effects involving context order $(p s>.15)$. 


\section{Oxygenation}

400

Figure 3 shows the timecourse of oxygenation in each PFC subregion for all conditions

401 (averaged across participants). Looking at these timecourses, it is evident that oxygenation is

402 fairly stable over time. The main exception is the first 10-30 s of each condition, which is

403 approximately the time it takes for the hemodynamic response to evolve in response to the

404 demands of the task. This lag provides some validation for our decision to exclude the first $10 \mathrm{~s}$

405 of data when calculating block means (see Data Processing). The increase in oxygenation at the

406 very start of each block may reflect the increase in cingulo-opercular network activity that

407 accompanies task onset (Eckert et al. 2016), which may in turn trigger the activation of brain

408 areas that we are able to measure (e.g., the DLPFC and IFG). For the easy conditions,

409 oxygenation then decreases, hitting a trough after the $50 \mathrm{~s}$ mark. It then ramps up again until 150

410 s, at which point it plateaus for the remainder of the block. This may reflect an ongoing

411 recalibration of cognitive resources to meet the relatively low task demands without recruiting

412 more than is necessary. In contrast, for the hard conditions, oxygenation remains relatively high

413 and steady throughout, likely reflecting their higher task demands. 

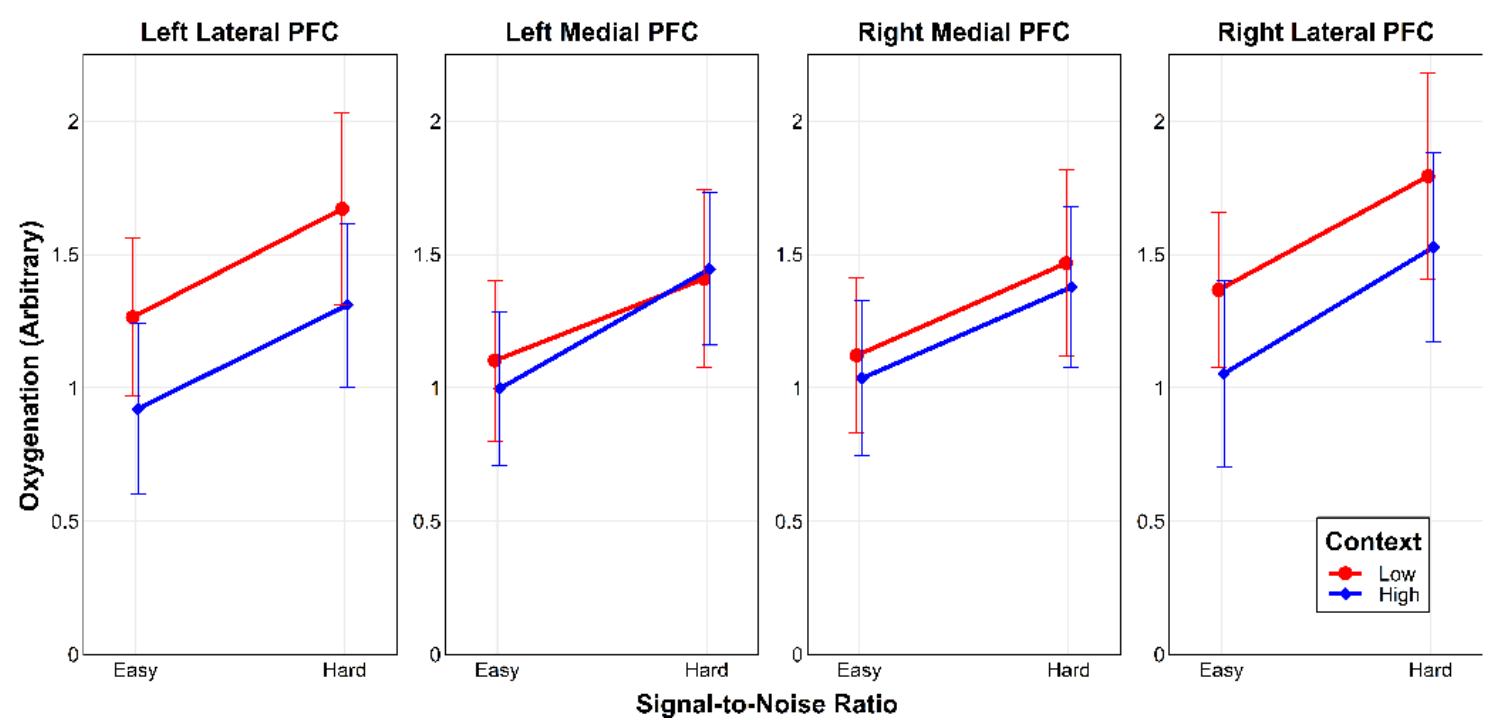

417 Figure 3. Oxygenation in all four PFC subregions was fairly stable over time in each block, with

418 the exception of the first 10-30 s over which oxygenation started to evolve in response to task

419 demands. After this period, differences between blocks become readily apparent. Oxygenation is

420 in arbitrary units, since continuous wave fNIRS is unable to measure the absolute concentration

421 of oxygen. The zero mark on the y-axis indicates the level of oxygenation at baseline.

422

A repeated-measures $2(\mathrm{SNR}) \times 2($ context $) \times 4(\mathrm{PFC}$ subregion $)$ found a significant

main effect of SNR on oxygenation $\left(F[1,27]=26.9, p<.001, \eta_{\mathrm{G}}{ }^{2}=.014\right)$, with oxygenation

424

greater when the SNR was hard than when the SNR was easy. There was no effect of context

425

$\left(F[1,27]=1.66, p=.21, \eta_{\mathrm{G}}{ }^{2}=.003\right), \mathrm{PFC}$ subregion $\left(F[3,81]=0.36, p=.68, \eta_{\mathrm{G}}{ }^{2}=.002\right), \mathrm{SNR} \times$

context $\left(F[1,27]=0.043, p=.84, \eta_{\mathrm{G}}^{2}=.00004\right)$, or $\mathrm{SNR} \times$ context $\times$ PFC subregion $(F[3,81]=$

427

$\left.0.63, p=.57, \eta_{\mathrm{G}}{ }^{2}=.00009\right)$ on oxygenation. Although the effect of context $\times$ PFC subregion on

428

oxygenation was not significant, it trended toward significance $\left(F[3,81]=3.05, p=.050, \eta_{\mathrm{G}}{ }^{2}=\right.$

429

.002), suggesting that the effect of context may have differed by PFC subregion. Given our

430

prediction that oxygenation in only the left lateral PFC would show an effect of context (as well 
Figure 4 shows mean oxygenation in each PFC subregion as a function of SNR and

433

434

435

436

437

438

439

440

441

442

443

context (averaged across participants and time within each block). We analyzed oxygenation in each PFC subregion separately using repeated-measures $2(\mathrm{SNR}) \times 2$ (context) ANOVAs. In each PFC subregion, there was a significant effect of SNR on oxygenation $(p s<.001)$, with oxygenation greater when the SNR was hard. There was a significant effect of context on oxygenation in the left lateral $\operatorname{PFC}\left(F[1,27]=4.87, p=.036, \eta_{\mathrm{G}}{ }^{2}=.011\right)$, with oxygenation greater when context was low. This effect of context was not present in the other PFC subregions ( $p$ s $>.17)$. SNR did not interact with context in any PFC subregion $(p s>.55)$. Given that oxygenation often continued to evolve for longer than just 10 s (see Figure 3), we also reanalyzed each PFC subregion after cutting off the first $30 \mathrm{~s}$ of each block. This re-analysis yielded the same results: a significant effect of SNR in each PFC subregion $(p s<.001)$ and an effect of context in only the left lateral PFC $\left(F[1,27]=4.71, p=.038, \eta_{\mathrm{G}}{ }^{2}=.011\right)$.

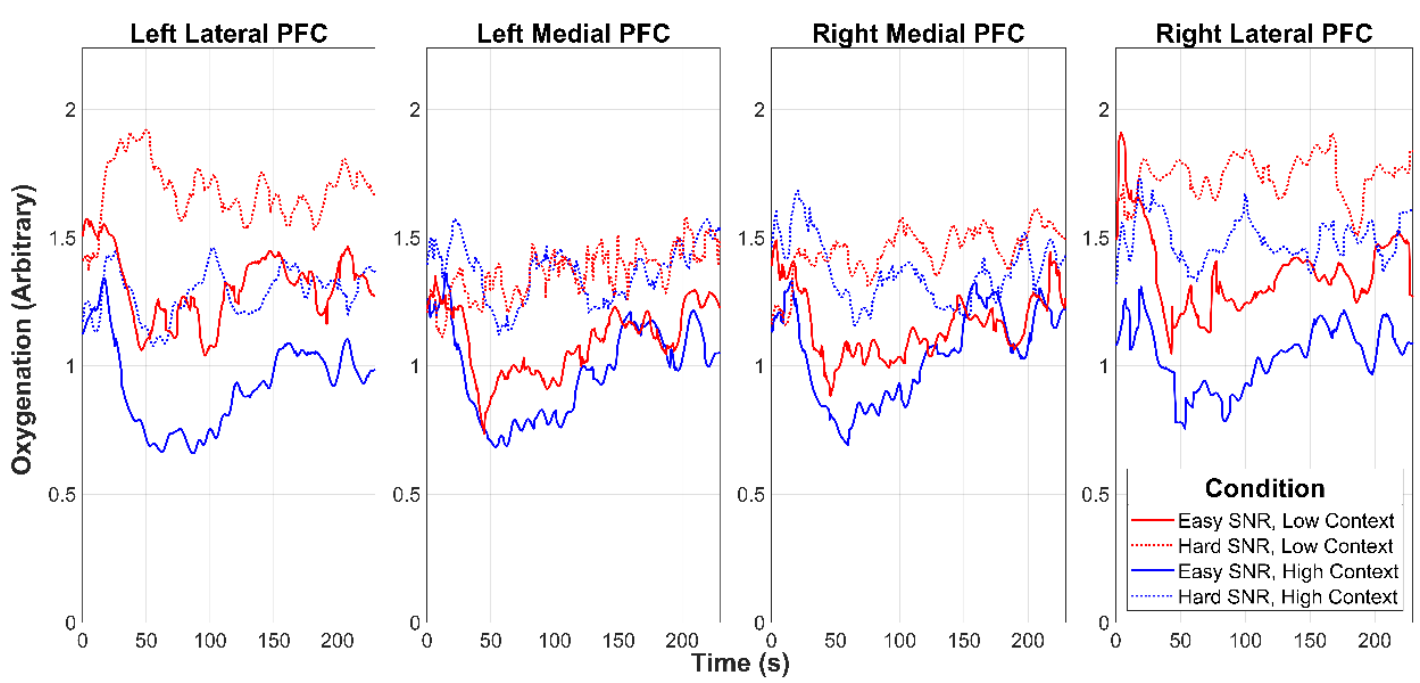

Figure 4. Oxygenation in all four PFC subregions was greater when the SNR was hard than when the SNR was easy, but only the left lateral PFC oxygenation was reduced when context was high. Error bars show the standard errors of the mean. 
As with accuracy, we explored whether oxygenation in each PFC subregion may have been affected by context order using a mixed 2 (SNR; within-subject) $\times 2$ (context; withinsubject $) \times 2$ (context order; between-subject) ANOVA. There was no effect of context $\times$ context order on oxygenation in the left lateral $\operatorname{PFC}\left(F[1,26]=0.067, p=.80, \eta_{\mathrm{G}}{ }^{2}=.0002\right)$, left medial $\operatorname{PFC}\left(F[1,26]=0.050, p=.83, \eta_{\mathrm{G}}^{2}=.0001\right)$, right medial $\operatorname{PFC}\left(F[1,26]=0.099, p=.76, \eta_{\mathrm{G}}^{2}=\right.$ $.0002)$, or right lateral PFC $\left(F[1,26]=1.06, p=.31, \eta_{\mathrm{G}}^{2}=.003\right)$. However, there was a significant effect of SNR $\times$ context $\times$ context order on oxygenation in all PFC subregions but the right lateral $(p s<.043)$. Upon further exploration, these interactions appeared to be driven by larger effects of SNR in the first context level than the second level, regardless of whether that first level was low or high. This may be due to fatigue effects. There were no other significant effects involving context order in any PFC subregion $(p s>.092)$.

\section{Relationship Between Accuracy and Oxygenation}

Two exploratory analyses further probed the relationship between accuracy and PFC oxygenation. First, paired-samples $t$-tests found that accuracy did not differ between easy SNR/low context and hard SNR/high context conditions (in other words, the conditions in which one factor was favourable and the other was unfavourable; $t[27]=-0.95, p=.35, d=0.18$ ). Likewise, left lateral PFC oxygenation also did not differ between these two conditions $(t[27]=-$ $0.30, p=.77, d=0.06)$. Means were also very similar across these conditions for both accuracy (easy SNR/low context: $M=0.69, S D=0.13$; hard SNR/high context: $M=0.72, S D=0.20$ ) and left lateral PFC oxygenation (easy SNR/low context: $M=1.26, S D=1.57$; hard SNR/high context: $M=1.31, S D=1.62)$. Second, a repeated-measures correlational analysis found that accuracy was significantly negatively correlated with left lateral PFC oxygenation, meaning that across conditions, as accuracy became worse, left lateral PFC oxygenation increased $\left(r_{\mathrm{rm}}[83]=-\right.$ 
$471.37, p<.001)$. Although not quite as strong, this negative correlation was also present in the right

472 lateral PFC $\left(r_{\mathrm{rm}}[83]=-.27, p=.011\right)$ and right medial PFC $\left(r_{\mathrm{rm}}[83]=-.25, p=.022\right)$, but it did

473 not reach significance in the left medial PFC $\left(r_{\mathrm{rm}}[83]=-.20, p=.063\right)$.

\section{Discussion}

475 Speech Understanding (Accuracy)

476

477

478

479

480

481

482

483

484

485

486

487

488

489

490

491

492

493
We found that when the SNR was decreased, accuracy on the R-SPIN Test became worse. In other words, as the level of background noise increased relative to speech, speech understanding deteriorated. Furthermore, speech understanding also deteriorated when sentences contained less semantic context (i.e., when the final word was less predictable). These results are consistent with a large body of research (Kalikow et al. 1977; Sarampalis et al. 2009; Wilson et al. 2012; Johnson et al. 2015) and demonstrate that the acoustic challenge of speech (e.g., the SNR) as well as the linguistic challenge of speech (e.g., the level of context) can both influence speech understanding. In addition, we found that context had a greater positive impact on speech understanding when the SNR was hard. This is consistent with Pichora-Fuller et al. (1995), who concluded that if masking is not severe enough, speech can be sufficiently well understood using lower-level processing, which results in a smaller effect of context.

\section{Listening Effort (Oxygenation)}

Effect of SNR on Listening Effort. In line with our prediction, we found that when the SNR was decreased, left lateral PFC oxygenation increased. In other words, as the level of background noise increased, participants exerted more listening effort. This is consistent with a large body of research, including studies using subjective (Rudner et al. 2012), behavioural (Sarampalis et al. 2009; Houben et al. 2013), peripheral physiological (Zekveld et al. 2010), and neural measures (Scott et al. 2004; Du et al. 2014). For instance, Du et al. (2014) reported 
494 increased activation of the left IFG (among other brain areas) as the level of broadband noise 495 increased. Although prior studies employing fNIRS have found effects of noise vocoding on 496 listening effort (Wijayasiri et al. 2017), the current study is the first to show an effect of SNR on 497 listening effort. This is in contrast to Rowland et al. (2018), an fNIRS study that did not find this 498 effect. The discrepancy in findings across the two studies may be due to the considerable 499 variability in the stimuli that Rowland et al. (2018) used; for instance, their noise was taken from 50017 different real-world environments (e.g., a busy café, a swimming pool, a moving car). In 501 contrast, the stimuli used in the current study were relatively well controlled. The left lateral PFC subregion may have been more active at the harder SNR to support processes such as stream segregation, selective attention, and perceptual closure, all of which

504 help listeners overcome masking from background noise (Mattys et al. 2012; Johnsrude \& Rodd 505 2016). This subregion includes the left DLPFC, part of the multiple-demand system, and the left

506 IFG, part of the speech processing network (Liu et al. 2017). The DLPFC was likely recruited to 507 implement cognitive control by selectively attending to the target speech and suppressing 508 distracting noise (Eckert et al. 2016). In addition, the IFG may have contributed verbal working 509 memory, which would allow any unclear aspects of the sentences (e.g., the final word) to be held 510 in memory for further processing (Peelle \& Wingfield 2016; Peelle 2018). Such further 511 processing could include two other proposed functions of the speech processing network:

512 predicting the sensory consequences of articulatory gestures (Hervais-Adelman et al. 2012; Du et 513 al. 2016) and higher-level linguistic processing. In the case of the latter function, the IFG likely 514 used prior linguistic knowledge to support perceptual closure (Davis \& Johnsrude 2003; Wild et 515 al. 2012). For instance, if noise occluded a portion of the final word in the sentence "Tom could 516 have thought about the -ort," our knowledge of words and syntax could help us arrive at "sport" 
517 as a viable candidate, rather than a non-word such as "gort" or a word that is unlikely

518 syntactically such as "short” (Ganong 1980; Bashford et al. 1992).

Interestingly, as the SNR decreased, oxygenation increased across all four subregions of

520 the PFC, not just the left lateral PFC. This result is not likely due to the limited spatial resolution

521 of fNIRS, since our context manipulation in the current study, as well as other manipulations in

522 our prior research (Rovetti et al. 2021), have used the same fNIRS apparatus to find effects

523 localized to specific PFC subregions. It is not uncommon for studies of effortful listening to

524 report activation of the right DLPFC and IFG (Salvi et al. 2002; Wong et al. 2008; Zekveld et al.

525 2014), which likely perform a similar function to their left-sided counterparts. In the case of the

526 IFG, it has been proposed that right-sided activation may reflect compensatory cognitive

527 resource recruitment to support speech understanding in especially challenging conditions

528 (Bidelman \& Howell 2016). Similarly, studies of effortful listening (Dimitrijevic et al. 2019) and

529 cognitive workload (Ayaz et al. 2012) have found activation of the medial PFC (e.g., frontopolar

530 cortex). This may also reflect compensatory activation in challenging conditions (Pochon et al.

531 2002). However, it should be noted that such compensation has yet to be well characterized in

532 the context of effortful listening (see Herrmann \& Johnsrude 2020).

Effect of Semantic Context on Listening Effort. In line with our prediction, we found

534 that when semantic context was lower, oxygenation in the left lateral PFC (but not other PFC

535 subregions) increased. In other words, as context rendered speech less predictable, listening

536 effort increased. This is consistent with some prior studies using subjective (Holmes et al. 2018),

537 behavioural (Johnson et al. 2015), peripheral physiological (Kadem et al. 2020), and neural

538 measures of listening effort (Davis et al. 2011). For instance, Davis et al. (2011) found that

539 activation of the left IFG was greater when participants listened to sentences low in context, but 
540 only for SNRs of $-2 \mathrm{~dB}$ or easier. Given that $-2 \mathrm{~dB}$ was the hardest SNR that we used, their

541 results are broadly consistent with ours. The current study was the first to use fNIRS to assess the

542 effect of semantic context (or any other linguistic factor) on listening effort.

543 The left IFG may have been more active for low-context sentences to compensate for the

544 lack of contextual constraint, which in turn increases processing load (Vitello \& Rodd 2015;

545 Johnsrude \& Rodd 2016). This brain area has been proposed to resolve lexical competition by 546 using prior knowledge to make predictions about incoming speech, which are then compared to 547 acoustic representations of speech in the superior temporal gyrus (Sohoglu et al. 2012). Activity 548 of the (usually left) IFG has also been found to increase in response to other linguistic 549 challenges, such as semantic ambiguity (Bekinschtein et al. 2011), syntactic ambiguity (Tyler et 550 al. 2011), syntactic complexity (Obleser et al. 2011), and syntactic incorrectness (Herrmann et al. 551 2012). It is also possible that with less context to enable higher-level linguistic processing, this 552 may have increased the load on other forms of processing (Johnsrude \& Rodd 2016), including 553 those associated with the DLPFC such as cognitive control.

To understand the higher-level linguistic processing described above, recall that the 555 greater lexical competition of low-context sentences means that participants likely relied heavily 556 on their knowledge of words and syntax (Ganong 1980; Bashford et al. 1992), as these are some 557 of the only linguistic strategies that would support the comprehension of low-context sentences 558 (see Effect of SNR on Listening Effort). In contrast, for high-context sentences, participants 559 could also rely on conditional word frequencies and general knowledge of the word, as the rich 560 context renders such processing useful (Warren 1970; Bashford et al. 1992; Johnsrude \& Rodd 561 2016). For instance, if noise occlusion of the final word caused a sentence to be heard as "She 562 had to vacuum the -ug," this final word would be difficult to identify based solely on knowledge 
563 of words and syntax given the number of viable candidates (e.g., mug, bug, drug). However, with

564 the preceding context offer by the sentence, identifying the word ("rug") becomes trivial. In the

565 current study, the lexical search process may have thus been more effortful for low-context

566 sentences than high-context sentences, or perhaps it simply required more time, with more

567 cognitive resources recruited overall (Wagner et al. 2016a).

\section{Relationship Between Speech Understanding and Listening Effort}

569

570

571

572

573

574

575

576

577 in the left medial PFC. Speech understanding has previously been found to have this relationship

578 with non-neural measures of listening effort (Zekveld et al. 2010; Holmes et al. 2018; but see

579 Winn \& Peece 2020). This further suggests a compensatory response of the PFC as listening

580 conditions become more challenging (Davis \& Johnsrude 2003).

581

582

583

584

585

In an exploratory analysis, we found that speech understanding did not differ between the easy SNR/low context and hard SNR/high context conditions, suggesting that acoustic and linguistic factors can offset one another in support of speech understanding (Johnsrude \& Rodd 2016). Interestingly, this was also true for listening effort, which like speech understanding did not differ between easy SNR/low context and hard SNR/high context conditions. A second exploratory analysis found that as speech understanding deteriorated across conditions (i.e., within subjects rather than between subjects), left lateral PFC oxygenation (i.e., listening effort) increased. This relationship was also found in the right lateral PFC and right medial PFC, but not

\section{Limitations and Future Directions}

Some limitations of the current study relate to fNIRS and the specific apparatus employed. For instance, the limited spatial resolution of fNIRS means that we were not able to distinguish activation of the DLPFC and IFG. The specific apparatus used is also limited to measurement of the PFC. This, coupled with the penetration depth limits of fNIRS, meant that 
we were unable to measure other brain areas of interest beyond the PFC, such as the anterior cingulate cortex (Eckert et al. 2016), premotor cortex (Peelle et al. 2018), and inferior parietal lobule (Alain et al. 2018). In addition, the fNIRS apparatus used does not include short separation channels, which can account for blood flow in extracranial tissue (Brigadoi \& Cooper 2018). Event-related analyses were also not possible given the block design, and thus incorrect trials could not be analyzed separately from "pass" trials. Finally, our results may not generalize to other measures of listening effort, particularly self-reported listening effort (Herrmann \& Johnsrude 2020), given that different measures frequently fail to correlate with one another and are often proposed to measure different constructs (Strand et al. 2018; Alhanbali et al. 2019; Strand et al. 2020). Nevertheless, the lack of a convergent measure of listening effort may be considered a limitation of the current study, particularly a measure that, like fNIRS, is purported to measure the recruitment of cognitive resources to support listening (e.g., pupillometry).

In the future, studies should continue to assess the effect of semantic context on listening effort, as there is still much to be clarified. One problem is that studies have been highly variable in their methodology, including their stimuli and the measures of listening effort used, which may explain why they differ in their conclusions. For instance, the effect of context may depend on the nature of the sentences and how they are processed (Winn \& Peece 2020) or on the severity of masking (Davis et al. 2011; Johnson et al. 2015). When it comes to the measures of listening effort used, different approaches have disagreed about the effect of context (c.f. Desjardins \& Doherty 2014; Holmes et al. 2018). However, rather than genuinely disagreeing, it is possible that each of these measures reflects a different dimension of effortful listening (e.g., the recruitment of cognitive resources versus the subjective experience of effort). To address these concerns, future studies should consider using more diverse stimuli, a wider range of 
609 SNRs, and multiple measures, with the aim of determining the conditions under which context

610 reduces listening effort. Finally, future fNIRS studies could employ event-related designs

611 (Lawrence et al. 2021); more channels covering frontal, temporal, and parietal areas; as well as

612 short-separation channels subjected to general linear modeling to better account for physiological

613 noise.

614 Conclusions

615

616 including the left lateral PFC, increases as SNR decreases. It was also the first study to assess

617 whether semantic context affects PFC oxygenation, and indeed we found that oxygenation in the

618 left lateral PFC is greater when listening to sentences with little to no context, as opposed to

619 sentences rich in context. We interpret these results to mean that listening effort (i.e., the

620 recruitment of cognitive resources to support listening) increases when listening to speech with

621 greater background noise and less context, likely to compensate for the challenges that they

622 impose on speech perception. These results highlight the fact that speech intelligibility alone

623 does not offer a complete picture of one's listening experience, and that ease of processing can

624 be influenced by linguistic factors (e.g., context) in addition to acoustic factors (e.g., SNR). They

625 also support the utility of fNIRS to measure listening effort, and perhaps support its candidacy as

626 a clinical tool to gain a more complete picture of patients' hearing health.

\section{Acknowledgements}

J.R. designed the experiment, collected the data, processed the data, statistically analyzed 629 the data, created visualizations, wrote the original manuscript, and revised the manuscript; H.G.

630 designed the experiment and revised the manuscript; M.Z. processed the data, created

631 visualizations, and revised the manuscript; F.A.R. designed the experiment and revised the 
632 manuscript. The authors would like to thank Rebecca Nurgitz for her pilot work that made this 633 study possible, Fran Copelli for her help with setting up the study and creating visualizations, 634 and Dr. Kathy Pichora-Fuller for her helpful insights into the results.

635

636

637

638

639

640

641

642

643

644

645

646

647

648

649

650

651

652

653

654 
655

656

657

658

659

660

661

662

663

664

665

666

667

668

669

670

671

672

673

674

675

676

\section{References}

Adank, P., Davis, M. H., \& Hagoort, P. (2012). Neural dissociation in processing noise and accent in spoken language comprehension. Neuropsychologia, 50, 77-84. doi:10.1016/j.neuropsychologia.2011.10.024

Alain, C., Du, Y., Bernstein, L. J., Barten, T., \& Banai, K. (2018). Listening under difficult conditions: An activation likelihood estimation meta-analysis. Human Brain Mapping, 39, 2695-2709. doi:10.1002/hbm.24031

Alhanbali, S., Dawes, P., Millman, R. E., \& Munro, K. J. (2019). Measures of listening effort are multidimensional. Ear and Hearing, 40, 1084-1097. doi:10.1097/aud.0000 000000000697

Anderson, A. A., Parsa, K., Geiger, S., Zaragoza, R., Kermanian, R., Miguel, H., Dashtestani, H., Chowdhry, F. A., Smith, E., Aram, S., Gandjbakhche, A. H. (2018). Exploring the role of task performance and learning style on prefrontal hemodynamics during a working memory task. PLOS One, 13, 1-16. doi:10.1371/journal.pone.0198257

Aranyi, G., Charles, F., \& Cavazza, M. (2015). Anger-based BCI using fNIRS neurofeedback. Proceedings of the 28th Annual ACM Symposium on User Interface Software \& Technology, 511-521. doi:10.1145/2807442.2807447

Ayaz, H., Onaral, B. (2005). Analytical software and stimulus-presentation platform to utilize, visualize and analyze near-infrared spectroscopy measures (Unpublished master's thesis). Drexel University, Philadelphia, PA.

Ayaz, H., Izzetoglu, M., Shewokis, P. A., \& Onaral, B. (2010). Sliding-window motion artifact rejection for functional near-infrared spectroscopy. Conference proceedings of the 
Annual International Conference of the IEEE Engineering in Medicine and Biology Society, 6567-6570. doi:10.1109/IEMBS.2010.5627113.

Ayaz, H., Shewokis, P. A., Bunce, S., Izzetoglu, K., Willems, B., \& Onaral, B. (2012). Optical brain monitoring for operator training and mental workload assessment. NeuroImage, 59, 36-47. doi:10.1016/j.neuroimage.2011.06.023

Borghini, G., \& Hazan, V. (2020). Effects of acoustic and semantic cues on listening effort during native and non-native speech perception. The Journal of the Acoustical Society of America, 147, 3783-3794. doi:10.1121/10.0001126

Bakdash, J. Z., \& Marusich, L. R. (2017). Repeated measures correlation. Frontiers in Psychology, 8, 1-13. doi:10.3389/fpsyg.2017.00456

Bashford, J.A., Riener, K.R., \& Warren, R.M. (1992). Increasing the intelligibility of speech through multiple phonemic restorations. Perception \& Psychophysics, 51, 211-217. doi:10.3758/bf03212247

Bekinschtein, T. A., Davis, M. H., Rodd, J. M., \& Owen, A. M. (2011). Why clowns taste funny: The relationship between humor and semantic ambiguity. Journal of Neuroscience, 31, 9665-9671. doi:10.1523/jneurosci.5058-10.2011

Bidelman, G.M., Howell, M. (2016). Functional changes in inter- and intra-hemispheric cortical processing underlying degraded speech perception. NeuroImage, 124, 581-590. doi:10.1016/j.neuroimage.2015.09.020

Bilger, R. C., Nuetzel, J. M., Rabinowitz, W. M., \& Rzeczkowski, C. (1984). Standardization of a test of speech perception in noise. Journal of Speech Language and Hearing Research, 27, 32-48. doi:10.1044/jshr.2701.32 
Brigadoi, S., \& Cooper, R.J. (2015). How short is short? Optimum source-detector distance for short-separation channels in functional near-infrared spectroscopy. Neurophotonics, 2(2), 1-9. doi:10.1117/1.NPh.2.2.025005

Brungart, D. S. (2001). Informational and energetic masking effects in the perception of two simultaneous talkers. The Journal of the Acoustical Society of America, 109, 11011109. doi:10.1121/1.1345696

Camilleri, J. A., Müller, V. I., Fox, P., Laird, A. R., Hoffstaedter, F., Kalenscher, T., \& Eickhoff, S. B. (2018). Definition and characterization of an extended multiple-demand network. NeuroImage, 165, 138-147. doi:10.1016/j.neuroimage.2017.10.020

Cavazza M., Aranyi G., Charles F. (2015) Brain-computer interfacing to heuristic search: First results. In: Ferrández Vicente J., Álvarez-Sánchez J., de la Paz López F., Toledo-Moreo F., Adeli H. (Eds.). Lecture Notes in Computer Science: Vol. 9107. Artificial Computation in Biology and Medicine (pp. 312-321). doi:10.1007/978-3-319-18914-7_33

Cui, X., Bray, S., Bryant, D. M., Glover, G. H., \& Reiss, A. L. (2011). A quantitative comparison of NIRS and fMRI across multiple cognitive tasks. NeuroImage, 54, 28082821. doi:10.1016/j.neuroimage.2010.10.069

Dahan, D., \& Tanenhaus, M. K. (2004). Continuous mapping from sound to meaning in spoken-language comprehension: Immediate effects of verb-based thematic constraints. Journal of Experimental Psychology: Learning, Memory, and Cognition, 30, 498-513. doi:10.1037/0278-7393.30.2.498

Davis, M. H., Ford, M. A., Kherif, F., \& Johnsrude, I. S. (2011). Does semantic context benefit speech understanding through "top-down" processes? Evidence from time-resolved 
sparse fMRI. Journal of Cognitive Neuroscience, 23, 3914-3932.

doi:10.1162/jocn_a_00084

Davis, M. H., \& Johnsrude, I. S. (2003). Hierarchical processing in spoken language comprehension. The Journal of Neuroscience, 23, 3423-3431. doi:10.1523/jneurosci.23-08-03423.2003

Desjardins, J. L., \& Doherty, K. A. (2014). The effect of hearing aid noise reduction on listening effort in hearing-impaired adults. Ear and Hearing, 35, 600-610. doi:10.1097/aud.0000000000000028

Dimitrijevic, A., Smith, M. L., Kadis, D. S., \& Moore, D. R. (2019). Neural indices of listening effort in noisy environments. Scientific Reports, 9, 1-10. doi:10.1038/s41598-019-47643-1

Dosenbach, N. U. F., Visscher, K. M., Palmer, E. D., Miezin, F. M., Wenger, K. K., Kang, H. C., E.D., Grimes, A.L., Schlaggar, B.L., \& Petersen, S. E. (2006). A core system for the implementation of task sets. Neuron, 50, 799-812. doi:10.1016/j.neuron.2006.04.031

Dosenbach, N. U. F., Fair, D. A., Cohen, A. L., Schlaggar, B. L., \& Petersen, S. E. (2008). A dual-networks architecture of top-down control. Trends in Cognitive Sciences, 12, 99105. doi:10.1016/j.tics.2008.01.001

Du, Y., Buchsbaum, B. R., Grady, C. L., \& Alain, C. (2014). Noise differentially impacts phoneme representations in the auditory and speech motor systems. Proceedings of the National Academy of Sciences, 111, 7126-7131. doi:10.1073/pnas.1318738111

Durlach, N. (2006). Auditory masking: Need for improved conceptual structure. The Journal of the Acoustical Society of America, 120, 1787-1790. doi:10.1121/1.2335426 
744 Duncan, J. (2010). The multiple-demand (MD) system of the primate brain: mental programs for

745

746

747

748

749

750

751

752

753

754

755

756

757

758

759

760

761

762

763

764 intelligent behaviour. Trends in Cognitive Sciences, 14, 172-179. doi:10.1016/j.tics.2010.01.004

Eckert, M. A., Menon, V., Walczak, A., Ahlstrom, J., Denslow, S., Horwitz, A., \& Dubno, J. R. (2009). At the heart of the ventral attention system: The right anterior insula. Human Brain Mapping, 30, 2530-2541. doi:10.1002/hbm.20688

Eckert, M. A., Teubner-Rhodes, S., \& Vaden, K. I. (2016). Is listening in noise worth It? The neurobiology of speech recognition in challenging listening conditions. Ear and Hearing, 37, 101S-110S. doi:10.1097/aud.0000000000000300

Eckert, M. A., Walczak, A., Ahlstrom, J., Denslow, S., Horwitz, A., \& Dubno, J. R. (2008). Agerelated effects on word recognition: Reliance on cognitive control systems with structural declines in speech-responsive cortex. Journal of the Association for Research in Otolaryngology, 9, 252-259. doi:10.1007/s10162-008-0113-3

Fishburn, F. A., Norr, M. E., Medvedev, A. V., \& Vaidya, C. J. (2014). Sensitivity of fNIRS to cognitive state and load. Frontiers in Human Neuroscience, 8, 1-11. doi:10.3389/fnhum.2014.00076

Fletcher, H., \& Galt, R.H. (1950). The perception of speech and its relation to telephony. The Journal of the Acoustical Society of America, 22, 89-151. doi:10.1121/1.1906605

Ganong, W.F. (1980). Phonetic categorization in auditory word perception. Journal of Experimental Psychology: Human Perception and Performance, 6, 110-125. doi:10.1037/0096-1523.6.1.110 
Harrivel, A. R., Weissman, D. H., Noll, D. C., \& Peltier, S. J. (2013). Monitoring attentional state with fNIRS. Frontiers in Human Neuroscience, 7, 1-10. doi:10.3389/fnhum.2013.00861

Herrmann, B., Obleser, J., Kalberlah, C., Haynes, J.-D., \& Friederici, A. D. (2011). Dissociable neural imprints of perception and grammar in auditory functional imaging. Human Brain Mapping, 33, 584-595. doi:10.1002/hbm.21235

Herrmann, B., \& Johnsrude, I. S. (2020). A model of listening engagement (MoLE). Hearing Research, 397, 1-12. doi:10.1016/j.heares.2020.108016

Hervais-Adelman, A. G., Carlyon, R. P., Johnsrude, I. S., \& Davis, M. H. (2012). Brain regions recruited for the effortful comprehension of noise-vocoded words. Language and Cognitive Processes, 27, 1145-1166. doi:10.1080/01690965.2012.662280

Hickok, G., \& Poeppel, D. (2007). The cortical organization of speech processing. Nature Reviews Neuroscience, 8, 393-402. doi:10.1038/nrn2113

Holmes, E., Folkeard, P., Johnsrude, I. S., \& Scollie, S. (2018). Semantic context improves speech intelligibility and reduces listening effort for listeners with hearing impairment. International Journal of Audiology, 57, 483-492. doi:10.1080/14992027.2018.1432901

Homan, R. W., Herman, J., \& Purdy, P. (1987). Cerebral location of international 10-20 system electrode placement. Electroencephalography and Clinical Neurophysiology, 66, 376382. doi:10.1016/0013-4694(87)90206-9

Houben, R., Doorn-Bierman, M. V., \& Dreschler, W. A. (2013). Using response time to speech as a measure for listening effort. International Journal of Audiology, 52, 753-761. doi:10.3109/14992027.2013.832415 
Izzetoglu, M. (2012). Functional optical brain imaging. In H. Liang, J. D. Bronzino, \& D. R. Peterson (Eds.), Biosignal Processing: Principles and Practices (pp. 1-34). Boca Raton FL: CRC Press.

Johnson, J., Xu, J., Cox, R., \& Pendergraft, P. (2015). A comparison of two methods for measuring listening effort as part of an audiologic test battery. American Journal of Audiology, 24, 419-431.doi:10.1044/2015_aja-14-0058

Johnsrude, I. S., \& Rodd, J. M. (2016). Factors that increase processing demands when listening to speech. In G. Hickok, \& S. L. Small (Eds.), Neurobiology of Language (pp. 491-502). Cambridge, MA: Elsevier Academic Press.

Kadem, M., Herrmann, B., Rodd, J., \& Johnsrude, I. S. (2020). Pupil dilation is sensitive to semantic ambiguity and acoustic degradation. doi:10.1101/2020.02.19.955609

Kato, T., Kamei, A., Takashima, S., Ozaki, T. (1993). Human visual cortical function during photic stimulation monitoring by means of near-infrared spectroscopy. Journal of Cerebral Blood Flow \& Metabolism, 13, 516-520. doi:10.1038/jcbfm.1993.66

Kocsis, L., Herman, P., \& Eke, A. (2006). The modified Beer-Lambert law revisited. Physics in Medicine and Biology, 51, N91-N98. doi:10.1088/0031-9155/51/5/n02

Kramer, S. E., Kapteyn, T. S., Festen, J. M., \& Kuik, D. J. (1997). Assessing aspects of auditory handicap by means of pupil dilatation. International Journal of Audiology, 36, 155-164. doi:10.3109/00206099709071969

Kuchinsky, S. E., Ahlstrom, J. B., Vaden, K. I., Cute, S. L., Humes, L. E., Dubno, J. R., \& Eckert, M. A. (2013). Pupil size varies with word listening and response selection difficulty in older adults with hearing loss. Psychophysiology, 50, 23-34. doi:10.1111/j.1469-8986.2012.01477.x 
811 Lakens, D., \& Caldwell, A. R. (2019). Simulation-based power-analysis for factorial

812

813 814

815

816

817

818

819

820

821

822

823

824

825

826

827

828

829

830

831

832

833 ANOVA designs. doi:10.31234/osf.io/baxsf

Lau, M. K., Hicks, C., Kroll, T., \& Zupancic, S. (2019). Effect of auditory task type on physiological and subjective measures of listening effort in individuals with normal hearing. Journal of Speech, Language, and Hearing Research, 62, 1549-1560. doi:10.1044/2018_jslhr-h-17-0473

Lawrence, R. J., Wiggins, I. M., Anderson, C. A., Davies-Thompson, J., \& Hartley, D. E. (2018). Cortical correlates of speech intelligibility measured using functional near-infrared spectroscopy (fNIRS). Hearing Research, 370, 53-64. doi:10.1016/j.heares.2018.09.005

Lawrence, R. J., Wiggins, I. M., Hodgson, J. C., \& Hartley, D. E. (2021). Evaluating cortical responses to speech in children: A functional near-infrared spectroscopy (fNIRS) study. Hearing Research, 401, 1-12. doi:10.1016/j.heares.2020.108155

Liang, L., Shewokis, P. A., \& Getchell, N. (2016). Brain activation in the prefrontal cortex during motor and cognitive tasks in adults. Journal of Behavioral and Brain Science, 6, 463-474. doi:10.4236/jbbs.2016.612042

Liu, Y., Piazza, E.A., Simony, E., Shewokis, P.A., Onaral, B., Hasson, U., \& Ayaz, H. (2017). Measuring speaker-listener neural coupling with functional near infrared spectroscopy. Scientific Reports, 7, 1-13. doi:10.1038/srep43293

Luce, P. A., \& Pisoni, D. B. (1998). Recognizing spoken words: The neighborhood activation model. Ear and Hearing, 19, 1-36. doi:10.1097/00003446-199802000-00001

Marek, S., \& Dosenbach, N.U.F. (2018). The frontoparietal network: function, electrophysiology, and importance of individual precision mapping. Dialogues in Clinical Neuroscience, 20,133-140. doi:10.31887/DCNS.2018.20.2/smarek 
study. Proceedings of the National Academy of Sciences, 99, 5669-5674. doi:10.1073/pnas.082111099

McCoy, S. L., Tun, P. A., Cox, L. C., Colangelo, M., Stewart, R. A., \& Wingfield, A. (2005). Hearing loss and perceptual effort: Downstream effects on older adults' memory for speech. The Quarterly Journal of Experimental Psychology Section A, 58, 22-33. doi:10.1080/02724980443000151

McGarrigle, R., Munro, K. J., Dawes, P., Stewart, A. J., Moore, D. R., Barry, J. G., \& Amitay, S. (2014). Listening effort and fatigue: What exactly are we measuring? A British Society of Audiology Cognition in Hearing Special Interest Group ‘white paper’. International Journal of Audiology, 53, 433-445. doi:10.3109/14992027.2014.890296

McClelland, J. L., \& Elman, J. L. (1986). The TRACE model of speech perception. Cognitive Psychology, 18, 1-86. doi:10.1016/0010-0285(86)90015-0

Metzger, F. G., Ehlis, A., Haeussinger, F. B., Schneeweiss, P., Hudak, J., Fallgatter, A. J., \& Schneider, S. (2017). Functional brain imaging of walking while talking - An fNIRS study. Neuroscience, 343, 85-93. doi:10.1016/j.neuroscience.2016.11.032

Miezin, F.M., Maccotta, L., Ollinger, J.M., Petersen, S.E., Buckner, \& R.L. (2000). Characterizing the hemodynamic response: Effects of presentation rate, sampling procedure, and the possibility of ordering brain activity based on relative timing. NeuroImage, 11, 735-759. doi:10.1006/nimg.2000.0568

Montgomery, C., Fisk, J. E., \& Roberts, C. A. (2017). Updating of working memory in ecstasy polydrug users: Findings from fNIRS. Human Psychopharmacology: Clinical and Experimental, 32, 1-12. doi:10.1002/hup.2609 
856

857

858

859

860

861

862

863

864

865

866

867

868

869

870

871

872

873

874

875

876

877

878

Moore, T. M., \& Picou, E. M. (2018). A potential bias in subjective ratings of mental effort. Journal of Speech Language and Hearing Research, 61, 1-17. doi:10.1044/2018_jslhr-h-17-0451

Obleser, J., Meyer, L., \& Friederici, A. D. (2011). Dynamic assignment of neural resources in auditory comprehension of complex sentences. NeuroImage, 56, 2310-2320. doi:10.1016/j.neuroimage.2011.03.035

Obleser, J., Wise, R. J. S., Alex Dresner, M., \& Scott, S. K. (2007). Functional integration across brain regions improves speech perception under adverse listening conditions. Journal of Neuroscience, 27, 2283-2289. doi:10.1523/jneurosci.4663-06.2007

Ohlenforst, B., Zekveld, A. A., Jansma, E. P., Wang, Y., Naylor, G., Lorens, A., Lunner, T., \& Kramer, S. E. (2017). Effects of hearing impairment and hearing aid amplification on listening effort. Ear and Hearing, 38, 267-281. doi:10.1097/aud.0000000000000396

Owen, A. M., Mcmillan, K. M., Laird, A. R., \& Bullmore, E. (2005). N-back working memory paradigm: A meta-analysis of normative functional neuroimaging studies. Human Brain Mapping, 25, 46-59. doi:10.1002/hbm.20131

Peelle, J. E. (2018). Listening Effort: How the cognitive consequences of acoustic challenge are reflected in brain and behavior. Ear and Hearing, 39, 204-214. doi:10.1097/aud.0000000000000494

Peelle, J. E., Johnsrude, I.S., \& Davis, M.H. (2010). Hierarchical processing for speech in human auditory cortex and beyond. Frontiers in Human Neuroscience, 4(51), 1-3. doi:10.3389/fnhum.2010.00051

Pichora-Fuller, M. K., Kramer, S. E., Eckert, M. A., Edwards, B., Hornsby, B. W., Humes, L. E., Lemke, U., Lunner, T., Matthen, M., Mackersie, C. L., Naylor, G., Phillips, N. A., 
Richter, M., Rudner, M., Sommers, M. S., Tremblay, K.L., Wingfield, A. (2016). Hearing impairment and cognitive energy: The Framework for Understanding Effortful Listening (FUEL). Ear \& Hearing, 37, 5S-27S. doi:10.1097/AUD.0000000000000312

Pichora-Fuller, M., Mick, P., \& Reed, M. (2015). Hearing, cognition, and healthy aging: Social and public health implications of the links between age-related declines in hearing and cognition. Seminars in Hearing, 36, 122-139. doi:10.1055/s-0035-1555116

Pichora-Fuller, M. K., Schneider, B. A., \& Daneman, M. (1995). How young and old adults listen to and remember speech in noise. The Journal of the Acoustical Society of America, 97, 593-608. doi:10.1121/1.412282

Pinti, P., Scholkmann, F., Hamilton, A., Burgess, P., \& Tachtsidis, I. (2019). Current status and issues regarding pre-processing of fNIRS neuroimaging data: An investigation of diverse signal filtering methods within a general linear model framework. Frontiers in Human Neuroscience, 12, 1 - 21. doi:10.3389/fnhum.2018.00505

Plack, C. J., Barker, D., \& Prendergast, G. (2014). Perceptual consequences of "hidden" hearing loss. Trends in Hearing, 18, 1-11. doi:10.1177/2331216514550621

Pochon, J. B., Levy, R., Fossati, P., Lehericy, S., Poline, J. B., Pillon, B., Le Bihan, D., Dubois, B. (2002). The neural system that bridges reward and cognition in humans: An fMRI study. Proceedings of the National Academy of Sciences, 99, 5669-5674. doi:10.1073/pnas.082111099

Ptak, R. (2011). The frontoparietal attention network of the human brain. The Neuroscientist, 18, 502-515. doi:10.1177/1073858411409051

R Core Team (2020). R: A language and environment for statistical computing. R Foundation for Statistical Computing, Vienna, Austria. Retrieved from https:/www.R-project.org/ 
902 903

904

905

906

907

908

909

910

911

912

913

914

915

916

917

918

919

920

921

922

923

Rodd, J. M., Davis, M. H., \& Johnsrude, I. S. (2005). The neural mechanisms of speech comprehension: fMRI studies of semantic ambiguity. Cerebral Cortex, 15, 1261-1269. doi:10.1093/cercor/bhi009

Rönnberg, J., Lunner, T., Zekveld, A., Sörqvist, P., Danielsson, H., Lyxell, B., Dahlström, Ö., Signoret, C., Stenfelt, S., Pichora-Fuller, M. K., Rudner, M. (2013). The Ease of Language Understanding (ELU) model: Theoretical, empirical, and clinical advances. Frontiers in Systems Neuroscience, 7, 1-17. doi:10.3389/fnsys.2013.00031

Rovetti, J., Goy, H., Pichora-Fuller, M. K., \& Russo, F. A. (2019). Functional near-infrared spectroscopy as a measure of listening effort in older adults who use hearing aids. Trends in Hearing, 23, 1-22. doi:10.1177/2331216519886722

Rovetti, J., Goy, H., Nurgitz, R., \& Russo, F. A. (2021). Comparing auditory and visual verbal working memory load using functional near-infrared spectroscopy. Behavioural Brain Research, 402, 1-10. doi:10.1016/j.bbr.2020.113102

Rowland, S. C., Hartley, D. E. H., \& Wiggins, I. M. (2018). Listening in naturalistic scenes: What can functional near-infrared spectroscopy and intersubject correlation analysis tell us about the underlying brain activity? Trends in Hearing, 22, 1-18. doi: $10.1177 / 2331216518804116$

Rudner, M., Lunner, T., Behrens, T., Thorén, E. S., \& Rönnberg, J. (2012). Working memory capacity may influence perceived effort during aided speech recognition in noise. Journal of the American Academy of Audiology, 23, 577-589. doi:10.3766/jaaa.23.7.7

Saleh, S., Sandroff, B.M., Vitiello, T., Owoeye, O., Hoxha, A., Hake, P., Goverover, Y., Wylie, G., Yue, G., DeLuca, J. (2018). The role of premotor areas in dual tasking in healthy 
controls and persons with multiple sclerosis: An fNIRS imaging study. Frontiers in Behavioral Neuroscience, 12, 1-11. doi:10.3389/fnbeh.2018.00296

Salvi, R. J., Lockwood, A. H., Frisina, R. D., Coad, M. L., Wack, D. S., \& Frisina, D. R. (2002). PET imaging of the normal human auditory system: Responses to speech in quiet and in background noise. Hearing Research, 170, 96-106. doi:10.1016/s0378-5955(02)00386-6

Sarampalis, A., Kalluri, S., Edwards, B., \& Hafter, E. (2009). Objective measures of listening effort: Effects of background noise and noise reduction. Journal of Speech Language and Hearing Research, 52, 1230-1240. doi:10.1044/1092-4388(2009/08-0111)

Scott, S. K., Rosen, S., Wickham, L., \& Wise, R. J. S. (2004). A positron emission tomography study of the neural basis of informational and energetic masking effects in speech perception. The Journal of the Acoustical Society of America, 115, 813-821. doi:10.1121/1.1639336

Sharp, D. J., Scott, S. K., Mehta, M. A., Wise, R.J.S. (2005). The neural correlates of declining performance with age: Evidence for age-related changes in cognitive control. Cerebral Cortex, 16, 1739-1749. doi:10.1093/cercor/bhj109

Skipper, J. I., Devlin, J. T., \& Lametti, D. R. (2017). The hearing ear is always found close to the speaking tongue : Review of the role of the motor system in speech perception. Brain and Language, 164, 77-105. doi:10.1016/j.bandl.2016.10.004

Sohoglu, E., Peelle, J. E., Carlyon, R. P., \& Davis, M. H. (2012). Predictive top-down integration of prior knowledge during speech perception. Journal of Neuroscience, 32, 8443-8453. doi:10.1523/jneurosci.5069-11.2012 
Strand, J., Simenstad, A., Cooperman, A., \& Rowe, J. (2013). Grammatical context constrains lexical competition in spoken word recognition. Memory \& Cognition, 42, 676-687. doi:10.3758/s13421-013-0378-6

Strand, J., Brown, V. A., Merchant, M. B., Brown, H. E., \& Smith, J. (2018). Measuring listening effort: Convergent validity, sensitivity, and links with cognitive and personality measures. Journal of Speech Language and Hearing Research, 61, 1-24. doi:10.1044/2018_jslhr-h-17-0257

Strand, J., Ray, L., Dillman-Hasso, N., \& Brown, V. A. (2020). Understanding speech amid the jingle and jangle: Recommendations for improving measurement practices in listening effort research. doi:10.31234/osf.io/3e7mf

Tun, P. A., McCoy, S., \& Wingfield, A. (2009). Aging, hearing acuity, and the attentional costs of effortful listening. Psychology and Aging, 24, 761-766. doi:10.1037/a0014802

Tyler, L. K., Marslen-Wilson, W. D., Randall, B., Wright, P., Devereux, B. J., Zhuang, J., Papoutsi, M., Stamatakis, E. A. (2011). Left inferior frontal cortex and syntax: Function, structure and behaviour in patients with left hemisphere damage. Brain, 134, 415-431. doi:10.1093/brain/awq369

Vaden, K. I., Kuchinsky, S. E., Cute, S. L., Ahlstrom, J. B., Dubno, J. R., \& Eckert, M. A. (2013). The cingulo-opercular network provides word-recognition benefit. Journal of Neuroscience, 33, 18979-18986. doi:10.1523/JNEUROSCI.1417-13.2013

Vaden, K. I., Kuchinsky, S. E., Ahlstrom, J. B., Dubno, J. R., \& Eckert, M. A. (2015). Cortical activity predicts which older adults recognize speech in noise and when. Journal of Neuroscience, 35, 3929-3937. doi:10.1523/jneurosci.2908-14.2015 
Van de Rijt, L., Opstal, A. J., Mylanus, E. A., Straatman, L. V., Hu, H. Y., Snik, A. F., \& Wanrooij, M. M. (2016). Temporal cortex activation to audiovisual speech in normalhearing and cochlear implant users measured with functional near-infrared spectroscopy. Frontiers in Human Neuroscience, 10, 1-14. doi:10.3389/fnhum.2016.00048

Vitello, S., \& Rodd, J.M. (2015). Resolving semantic ambiguities in sentences: Cognitive processes and brain mechanisms. Language and Linguistics Compass, 9, 391-405. doi:10.1111/lnc3.12160

Wagner, A., Toffanin, P., \& Başkent, D. (2016a). The timing and effort of lexical access in natural and degraded speech. Frontiers in Psychology, 7, 1-14. doi:10.3389/fpsyg.2016.00398

Wagner, A., Pals, C., de Blecourt, C.M., Sarampalis, A., \& Başkent, D. (2016b). Does signal degradation affect top-down processing of speech? Advances in Experimental Medicine and Biology, 894, 297-306. doi:10.1007/978-3-319-25474-6_31

Warren, R. M. (1970). Perceptual restoration of missing speech sounds. Science, 167, 392-393. doi:10.1126/science.167.3917.392

Wijayasiri, P., Hartley, D. E., \& Wiggins, I. M. (2017). Brain activity underlying the recovery of meaning from degraded speech: A functional near-infrared spectroscopy (fNIRS) study. Hearing Research, 351, 55-67. doi:10.1016/j.heares.2017.05.010

Wild, C. J., Yusuf, A., Wilson, D. E., Peelle, J. E., Davis, M. H., \& Johnsrude, I. S. (2012). Effortful listening: The processing of degraded speech depends critically on attention. Journal of Neuroscience, 32, 14010-14021. doi:10.1523/jneurosci.1528-12.2012 
Wilson, R. H., McArdle, R., Watts, K. L., \& Smith, S. L. (2012). The Revised Speech Perception in Noise Test (R-SPIN) in a multiple signal-to-noise ratio paradigm. Journal of the American Academy of Audiology, 23, 590-605. doi:10.3766/jaaa.23.7.9

Winn, M. (2016). Rapid release from listening effort resulting from semantic context, and effects of spectral degradation and cochlear implants. Trends in Hearing, 20, 1-17. doi:10.1177/2331216516669723

Winn, M., \& Teece, K. H. (2020). Listening effort is not the same as speech intelligibility score. doi:10.31234/osf.io/vk65w

Wisniewski, M.G., Thompson, E.R., Iyer, N., Estepp, J.R., Goder-Reiser, M.N., \& Sullivan, S.C. (2015). Frontal midline $\theta$ power as an index of listening effort. NeuroReport, 26, 94-99. doi:10.1097/WNR.0000000000000306

Wong, P. C. M., Uppunda, A. K., Parrish, T. B., \& Dhar, S. (2008). Cortical mechanisms of speech perception in noise. Journal of Speech Language and Hearing Research, 51, 1026-1041. doi:10.1044/1092-4388(2008/075)

Wu, Y.-H., Stangl, E., Zhang, X., Perkins, J., \& Eilers, E. (2016). Psychometric functions of dual-task paradigms for measuring listening effort. Ear and Hearing, 37, 660-670. doi:10.1097/aud.0000000000000335

Zekveld, A. A., Heslenfeld, D. J., Johnsrude, I. S., Versfeld, N. J., \& Kramer, S. E. (2014). The eye as a window to the listening brain: Neural correlates of pupil size as a measure of cognitive listening load. NeuroImage, 101, 76-86. doi:10.1016/j.neuroimage.2014.06.069

Zekveld, A. A., Kramer, S. E., \& Festen, J. M. (2010). Pupil response as an indication of effortful listening: The influence of sentence intelligibility. Ear and Hearing, 31, 480490. doi:10.1097/aud.0b013e3181d4f251 
1012 Zekveld, A. A., Rudner, M., Johnsrude, I. S., Heslenfeld, D. J., \& Rönnberg, J. (2012).

1013 Behavioral and fMRI evidence that cognitive ability modulates the effect of semantic 1014 context on speech intelligibility. Brain and Language, 122, 103-113.

1015 doi:10.1016/j.bandl.2012.05.006

1016 Zhou, X., Sobczak, G., McKay, C.M., \& Litovsky, R.Y. (accepted). Effortful listening to 1017 degraded speech and binaural unmasking resulting in release from effort investigated 1018 using functional near-infrared spectroscopy (fNIRS). Trends in Hearing. 